\title{
MCLP and SQM models for the emergency vehicle districting and location problem
}

\author{
Mohammad Mohammadi", Zeynab Dashti khotbesara and Abolfazl Mirzazadeh
}

Department of Industrial Engineering, Faculty of Engineering, Kharazmi University, Tehran, Iran

\begin{tabular}{l}
\hline C H R O N I C L E \\
\hline Article history: \\
Received March 14, 2014 \\
Accepted July 20, 2014 \\
Available online \\
July 21 2014 \\
\hline Keywords: \\
Location \\
Maximal covering location \\
problem (MCLP) \\
Spatial queuing model (SQM) \\
Simulated Annealing (SA) \\
Genetic Algorithm (GA) \\
Locate the emergency units \\
Metaheuristics
\end{tabular}

\section{A B S T R A C T}

\begin{abstract}
Over time, the number of unexpected earthy, oceanic and atmospheric events is rising each year. Hence, disaster management is considered as one of the most important scientific and practical issues in developed and developing countries. Therefore, in this study, we review and develop the problem of locating the emergency units with constraints including the number of available ambulances, limited budget for deployment of ambulances and the minimum acceptable level of covering. The proposed model improves the spatial queuing model (SQM) and Maximal Covering Location Problem (MCLP) by considering the cost of the deployment of the emergency units, which makes it closer to real-world conditions. Because the proposed model is NP-hard, the model is solved using three heuristics including Simulated Annealing (SA), Genetic Algorithm (GA) and a hybrid of both. The preliminary results indicate that the hybrid method had better performance to achieve the optimal or close to optimal solution.
\end{abstract}

(C) 2014 Growing Science Ltd. All rights reserved.

\section{Introduction}

Facility location (FL) is a 100-year history and some experts believe that it is a classic and ancient knowledge. Some experts believe that finding accommodations is a classical and ancient knowledge. The first FL model that was discussed and can be used is the Location Set Covering Model (LSCM) that was provided by Toregas et al. (1971) to aim of minimizing the number of ambulances needed to cover all the inquiries. This model is not very close to reality because the only criterion for locating units was the time of interval between the points and there were no differences between various demand points. Ball and Lin (1993) offered a reliable emergency location model. In this model, without considering the capacity of ambulances, a linear constraint to make the number of ambulances to reach a reliable level was added to the LSCM model. Church and Revelle (1974) presented Maximal Covering Location Problem model (MCLP). This model, because of the limited number of ambulances and specifying the points of demand, optimizes the services in circumstances, which would have the most surface coating. MCLP model is one of the most important of the positioning issues and usually it is the main base of other positioning models. The time for resolving MCLP model comply a non-polynomial function and these issues are said NP-hard issues (ReVelle,

* Corresponding author.

E-mail address: Mohammadi@khu.ac.ir (M. Mohammadi)

C 2014 Growing Science Ltd. All rights reserved.

doi: $10.5267 /$ j.ds1.2014.7.001 
1989). Pirkul and Schilling (1988) presented Capacitated Maximal Covering Location Problem model (CMCLP). This model, considered the capacity of ambulances, developed the MCLP model. The primary goal is the same as MCLP model and looks for the maximum total coverage of areas of requests. Shariff et al (2012) solved MCLP model with Genetic Algorithm. Maximal Expected Covering Location Problem model (MEXCLP) was presented by Daskin (1983) using the expansion of MCLP model. In this model, a probability $(q)$ is attributed to each ambulance, which represents unavailability of ambulance and the model was applied for a case study in city of Bangkok. They claimed that they could cover, in addition to increasing inquiries, and reduce the number of required ambulances from 21 to 15. This model was an extended model by Repede and Bernardo (1994) and they presented Time and Maximal Expected Covering Location Program model (TIMEXCLP) by considering the speed of traffic throughout the day. Fuzzy mode of MCLP model as well as was presented by Davari et al. (2011) and they solved the resulted problem using Simulated Annealing algorithm. Hogan and Revelle (1986) provided supporting coating model (BACOP). The fact that some regions need more ambulance due to their demands, in this model was emphasized. In this model by using double variable in MCLP model, at least, two ambulances were assigned for higherdemand areas. Gendreau et al. (1987) presented a Double Standard Model (DSM) model where a two-level coating was used. The purpose of this model maximized the requests for covered areas using two ambulances. Schmid and Doerner (2010) developed the double standard model. In 2012, they also considered a time period and the possibility of moving ambulances in various courses contributed DSM model and named multi period Double Standard Model (mDSM). Revelle and Hogan (1989) offered a probability model under the title Maximum Availability location Problem (MALP1). The model was to maximize covered requests with the probability of $\alpha$. In fact, a lack of access coefficient "q" was considered for relief centers. Locating model with optimization of two functions provided by Burkey et al. (2012). In this model, one of the target functions was to maximize coverage of inquiries. Locating models that has been raised in recent years can be Bottleneck Steiner tree model presented by Zhang et al. (2012). Syam and Cote (2010) presented a model to minimize fees and a spatial queuing model (SQM), which was originally presented by Geroliminis et al. (2009) for locations of emergency vehicles urban networks, with regard to the characteristics of the time and place inquiries. In SQM model, the probability of lack of availability of emergency units was considered and with this restriction it was closer to the real model. However, this model also has some weakness, that is the lack of possibility for establishment of more than one ambulance in a region, and decisions are only based on interval between time points and covers minimum level of areas but cannot improve the coating and maximize it according to the other limitations and conditions. Therefore, this paper presents a combination of MCLP model and SQM model by adding target function for minimizing the cost of establishment of units in each area.

\section{The proposed mathematical model}

In this model, our goal is to develop a distance line model and to solve the issue of positioning emergency medical or treatment units by considering the numbers limitations of the existing ambulances, budget and a minimum level of acceptable coating to minimize costs using target function. A decision maker determines the coefficients of the objective functions. In this model, unlike the SQM model, the possibility of establishing only one ambulance in each region is removed and it is possible to consider more than one ambulance. With the possibility of the establishment of several units, it is possible to reduce the cost of site construction. With establishment of one ambulance, with respect to response time, certain areas will be covered. In fact, we are interested in determining the place or places of deployment medical units with optimized response time, coverage area (Considering MCLP model) by optimizing the cost of construction and the establishment of units. By adding MCLP model to the proposed model, SQM model became more real and In addition to observance of the minimum level of coating, the possibility of achieving the optimum coating will be achieved. In addition, in the proposed model, the weakness of SQM model in considering the number of possible events as the sole criterion to determine demand for each region with a view to putting the severity of possible accidents and population density, somewhat repairable. 


\subsection{The model assumptions}

Assumptions and characteristics of the issue are determined as follows:

All of the parameters and data used in the issue are absolute.

There are $N$ separate ambulances.

No limited connections exist between them.

Ambulances across the schedule horizon, if not busy, are available.

Every ambulance in a moment can only be deployed to an area.

Demand in every area is depend on highly occurrence of events, number of events and population density.

All available ambulances are stationed.

There is a possibility of having no ambulance in some areas.

There is the possibility of the establishment of more than one ambulance at each location.

Area required for the deployment of ambulances has been considered identical.

Each ambulance can only be settled in one place.

The probability that at least one ambulance is available is $1-\left(1 / 2^{\wedge} \mathrm{N}\right) . \mathrm{N}$ is the number of the existing ambulances.

The symbols listed below are used for mathematical.

\subsection{Input Parameters}

$J$ : number of places or regions on demand,

$N$ : number of available ambulances,

$T_{n k j}$ : response time to demand location, $j$ when ambulance $n$ is based on the location $k$,

$A_{k}$ : land prices or the cost of the deployment of the units in the $k^{\text {th }}$ place,

$d$ : the area required for the establishment of any ambulance,

$\lambda_{\mathrm{j}}$ : Highly probable events at $\mathrm{j}$,

$\mathrm{h}_{\mathrm{j}}$ : The number of possible accidents at the location $\mathrm{j}$,

$M_{j}$ : Population density at the location $\mathrm{j}$,

$\mathrm{C}_{\text {cov }}$ : The minimum acceptable level of coverage,

$a$ : The minimum acceptable response time,

$B$ : available budget,

$\mathrm{w}_{\mathrm{m}}^{\prime}$ : Recommended weight $\mathrm{m}$ th target function used by user for model or system.

\subsection{Indices}

$n$ : the index number of the ambulance, $n=1, \ldots, N$,

$k$ : ambulance deployment locations index, $k=1, \ldots, J$,

$j$ : demand location index, $j=1, \ldots, J$,

$i$ : index of sites, $i=1, \ldots, I$,

$v, s$ : index of possible modes of response system, $v, s=1, \ldots, 2^{N}$,

$m$ : index of the number of available objective functions, $m=1,2,3$.

\subsection{Decision variables}

$Z$ : minimum cost deployment of ambulances,

$\mathrm{P}_{\mathrm{nkj}}$ : A fraction of the deployment to the area $j$ by $\mathrm{n}^{\text {th }}$ ambulance deployed in location $k$,

$\mathrm{x}_{\mathrm{ik}}$ : The number of ambulances in unit or base $i$, which are located on position $k$,

$\mathrm{y}_{j}:\{1$ if area $\mathrm{j}$ is coverd by at least one ambulance

$y_{j}:\left\{\begin{array}{ll}1 \\ 0\end{array}\right.$ if area $j$ is not coverd by any ambulance

$\mathrm{L}_{\mathrm{ik}}:\left\{\begin{array}{rr}1 & \text { if unit i is k locatin } \\ 0 & \text { else }\end{array}\right.$ 
$\mathrm{y}_{\mathrm{nkj}}^{\prime}:\left\{\begin{array}{c}1 \text { if area } \mathrm{j} \text { is covered by } \mathrm{n} \text { th ambulance } \\ \text { in k location }\end{array}\right.$,

$\mathrm{L}_{\mathrm{nk}}^{\prime}:\left\{\begin{array}{lr}1 & \text { if ambulance } \mathrm{n} \text { is in } \mathrm{k} \text { location } \\ 0 & \text { else }\end{array}\right.$,

$\mathrm{x}_{\mathrm{i}}$ : number of ambulances in base $i$,

$\mathrm{f}_{\mathrm{j}}$ : demand in $j$ area,

$\mathrm{w}_{\mathrm{kj}}$ : A set, which includes the database index,

$\mathrm{E}_{\mathrm{nkj}}$ : set of scenarios that serve $n$, which is the nearest available server in area $j$,

$\mathrm{B}_{\mathrm{s}}$ : Set of position of the ambulances in case $s \quad\left(\mathrm{~B}_{\mathrm{s}}=\left\{\mathrm{b}_{1}, \mathrm{~b}_{2}, \ldots, \mathrm{b}_{\mathrm{N}}\right\}\right)$,

$\mathrm{B}_{2^{\mathrm{N}}}$ : Set the position of the ambulances, when, no ambulance is available and idle,

$\mathrm{p}\left\{\mathrm{B}_{\mathrm{s}}\right\}$ : The possibility of partially related peak mode $\mathrm{B}_{\mathrm{s}}$,

$\mathrm{b}_{\mathrm{n}}: \begin{cases}1 & \text { if ambulance } \mathrm{n} \text { is buzy } \\ 0 & \text { else }\end{cases}$

$\mathrm{w}_{\mathrm{m}}: m^{\text {th }}$ coefficient of target function,

$\lambda_{\mathrm{sv}}$ and $\mu_{\mathrm{sv}}$ : is the upward and downward mean rates in which transitions are made from different states to state $v$ corresponding to vertices $B_{s}$ and $B_{v}$, given the system is in states. The decision variables are the number of ambulances and the establishment of units in each unit to minimize target function and apply desired limits.

\subsection{Proposed model}

The proposed model is formulated as follows:

Minimize $\mathrm{Z}=\mathrm{w}_{1} \sum_{\mathrm{n}=1}^{\mathrm{N}} \sum_{\mathrm{j}=1}^{\mathrm{J}} \sum_{\mathrm{k}=1}^{\mathrm{J}} \mathrm{L}_{\mathrm{nk}}^{\prime} \cdot \mathrm{P}_{\mathrm{nkj}} \cdot \mathrm{y}_{\mathrm{nkj}}^{\prime} \cdot \mathrm{t}_{\mathrm{nkj}}+\mathrm{w}_{2} \sum_{\mathrm{k}=1}^{\mathrm{J}} \sum_{\mathrm{i}=1}^{\mathrm{I}} \mathrm{L}_{\mathrm{ik}} \cdot \mathrm{A}_{\mathrm{k}} \cdot \mathrm{d} \cdot \mathrm{x}_{\mathrm{ik}}-\mathrm{w}_{3} \sum_{\mathrm{j}=1}^{\mathrm{J}} \mathrm{f}_{\mathrm{j}} \cdot \mathrm{y}_{\mathrm{j}}$

subject to

$f_{j}=\frac{\lambda_{j} \cdot h_{j} \cdot M_{j}}{\sum_{j=1}^{J} \lambda_{j} \cdot h_{j} \cdot M_{j}}$

$\mathrm{w}_{\mathrm{m}}=\frac{\mathrm{w}_{\mathrm{m}}}{\sum_{\mathrm{n}=1}^{3} \mathrm{w}_{\mathrm{n}}^{\prime}}, \forall \mathrm{m}=1,2,3$,

$\mathrm{x}_{\mathrm{i}}=\sum_{\mathrm{k}=1}^{\mathrm{J}} \mathrm{L}_{\mathrm{ik}} \cdot \mathrm{x}_{\mathrm{ik}}, \forall \mathrm{i}=1,2, \ldots, \mathrm{I}, \mathrm{I} \leq \mathrm{J}$,

$\sum_{j=1}^{J} f_{j} \cdot y_{j} \geq C_{c o v}$

$\sum_{i=1}^{I} x_{i}=N$

$\sum_{\mathrm{k}=1}^{\mathrm{J}} \sum_{\mathrm{i} \in \mathrm{w}_{\mathrm{kj}}} \mathrm{L}_{\mathrm{ik}} \cdot \mathrm{x}_{\mathrm{ik}} \geq \mathrm{y}_{\mathrm{j}}, \forall \mathrm{j}=1,2, \ldots, \mathrm{J}$,

$\mathrm{y}_{\mathrm{j}} \in\{0,1\}, \quad \forall \mathrm{j}=1,2, \ldots, \mathrm{J}$

$\mathrm{w}_{\mathrm{kj}}=\left\{\mathrm{i} \in \mathrm{I}, \mathrm{k} \in \mathrm{J} \mid \mathrm{t}_{\mathrm{ikj}} \leq \mathrm{a}\right\}$,

$\mathrm{P}_{\mathrm{nkj}}=\mathrm{f}_{\mathrm{j}} \cdot \frac{\sum_{\mathrm{B}_{\mathrm{s}} \in \mathrm{E}_{\mathrm{nkj}}} \mathrm{p}\left\{\mathrm{B}_{\mathrm{s}}\right\}}{\left(1-\mathrm{p}\left\{\mathrm{B}_{2^{\mathrm{N}}}\right\}\right)}, \forall \mathrm{j}, \mathrm{k}, \mathrm{n}$

$p\left\{B_{v}\right\}\left[\sum_{s_{\left\{B_{s} \in C_{N}: d_{s v}^{+}=1\right\}}} \lambda_{s v}+\sum_{s_{\left\{B_{s} \in C_{N}: d_{s v}^{-}=1\right\}}} \mu_{s v}\right]=\sum_{s_{\left\{B_{s} \in C_{N}: d_{v s}^{+}=1\right\}}} \lambda_{v s} p\left\{B_{s}\right\}+\sum_{s_{\left\{B_{s} \in C_{N}: d_{\overline{v s}}=1\right\}}} \mu_{v s} p\left\{B_{s}\right\} \quad\left(v=1,2, \ldots, 2^{N}\right)$ 


$$
\begin{aligned}
& \sum_{\mathrm{k}=1}^{J} \sum_{\mathrm{i}=1}^{\mathrm{I}} \mathrm{L}_{\mathrm{ik}} \cdot A_{\mathrm{k}} \cdot \mathrm{d} \cdot \mathrm{x}_{\mathrm{ik}} \leq \mathrm{B}, \\
& \sum_{\mathrm{s}=1}^{2^{\mathrm{N}}} \mathrm{p}\left\{\mathrm{B}_{\mathrm{s}}\right\}=1, \\
& \sum_{\mathrm{k}=1}^{J} \sum_{\mathrm{i}=1}^{\mathrm{I}} \mathrm{L}_{\mathrm{ik}}=\mathrm{I}, \\
& \sum_{\mathrm{i}=1}^{\mathrm{J}} \mathrm{L}_{\mathrm{ik}} \leq 1, \quad \forall \mathrm{k}=1,2, \ldots, \mathrm{J}, \\
& \sum_{\mathrm{j}=1}^{\mathrm{L}} \mathrm{L}_{\mathrm{ik}}=1, \quad \forall \mathrm{i}=1,2, \ldots, \mathrm{I}, \\
& \mathrm{y}_{\mathrm{nkj}}^{\prime} \in\{0,1\}, \forall \mathrm{j}, \mathrm{k}, \mathrm{n}, \\
& \mathrm{L}_{\mathrm{ik}} \in\{0,1\}, \forall \mathrm{k}=1,2, \ldots, \mathrm{J}, \forall \mathrm{i}=1,2, \ldots, \mathrm{I}, \\
& \mathrm{L}_{\mathrm{nk}}^{\prime} \in\{0,1\}, \forall \mathrm{k}=1,2, \ldots, \mathrm{J}, \forall \mathrm{n}=1,2, \ldots, \mathrm{N}
\end{aligned}
$$

Objective function in this model is composed of three target functions combined with specified coefficients. The three target functions minimize average response time, minimize cost of sites construction according to the number of ambulances and maximize surface coating of inquiries.

Constraints (1) determine the demand for each region (Based on population density, the number and severity of potential events). Constraints (2) specify the coefficients of each target function. Constraints (3) ensure that the number of ambulances managed in a certain area is exactly equal to the number specified for base. Constraints (4) is minimum coverage, which states that covering all area must be bigger than a predetermined amount $\left(\mathrm{C}_{\mathrm{cov}} \leq 1\right)$. Constraints (5) specify the number of facilities that should be deployed. Constraints (6) determine areas covered by acceptable distance, every area $j$ can only be covered if a facility exists in part of a place in time "a" if there is no such facilitate, left side of limits is zero and $y_{j}$ is forced to be zero. Constraints (7) are associated with decision variables, which must be zero or one. Constraints (8) represents a collection including $i$ base, which is located in place $k$ and cover $j$ area. Constraints (9) represent the fraction of dispatching ambulance $n$ to the area $j$ when located in area $k$. Constraints (10) are detailed balance equations determining steady-state probabilities of the "finite-state continuous time Markov process" model with $N$ servers. Constraints (11) ensure that the cost of the designated location would not exceed the available budget. Constraints (12) state that the sum of probabilities is equal one. The constraints (13) specify the number of sites, which must be established. Constraints (14) ensure that there is a maximum of one base is in any area. Constraints (15) ensure each health unit locate only in one place. Constraints (16), (17) and (18) ensure $L_{n k}^{\prime}, L_{i k}, y_{n k j}^{\prime}$ are zero or one. The upward transition rate, $\lambda_{\mathrm{ij}}$, can be considered as the demand for requested service that will create a transition from state $i$ to state $j$, given that the system is in state $i$, while the downward transition, $\mu_{\mathrm{ij}}$, is the service rate for this specific demand. In order to estimate upward and downward transition rates $\lambda_{\mathrm{ij}}$ and $\mu_{\mathrm{ij}}$, the term of $\mathrm{n}^{\text {th }}$ order districting is introduced. For $n=1$, the term is equivalent to districting as defined above; for $n$ $>1$, it is the partitioning of the study area in sub areas according to the $n^{\text {th }}$ nearest server. The $n^{\text {th }}$ order districting indicates that, for every region, the $\mathrm{n}_{1}$ nearest servers are unavailable and the nth responsible server will intervene if requested. Mathematically, this term can be expressed as follows:

$\mathrm{D}_{\mathrm{kl}}^{\mathrm{n}}=\left\{\mathrm{j} \in \mathrm{J}: \mathrm{t}_{\mathrm{lj}}=\min \left(\mathrm{n}, \mathrm{U}_{\mathrm{j}}\right)\right.$ and $\left.\mathrm{t}_{\mathrm{kj}}=\min \left(1, \mathrm{U}_{\mathrm{j}}\right)\right\}$, 
where $\mathrm{D}_{\mathrm{kl}}^{\mathrm{n}}$ is the sub-area belonging to the $1^{\text {st }}$ order district of server $k$ and the $n^{\text {th }}$ order district of server $l(\mathrm{k}=1,2, \ldots, \mathrm{N}$ and $\mathrm{l}=1,2, \ldots, \mathrm{N}) ; \min \left(\mathrm{n}, \mathrm{U}_{\mathrm{j}}\right)$ is the $n^{\text {th }}$ smaller term of the $\operatorname{set} \mathrm{U}_{\mathrm{j}}=\left\{\mathrm{t}_{\mathrm{ij}}\right\} \mathrm{i}=$ $1,2, \ldots, \mathrm{N} ; j$ is the service region; $J$ is the set of service regions in the service area.

For $n=1$, only districts $\mathrm{D}_{\mathrm{ii}}^{1}$ with $i=j$ can be defined and for $n>1$ only districts with $\mathrm{i} \neq \mathrm{j}$ make sense. Similarly, $\lambda_{\mathrm{kl}}^{\mathrm{n}}$ is defined as the total demand of the $\mathrm{D}_{\mathrm{kl}}^{\mathrm{n}}$ sub-area; it is straightforward that, for every order of districting, the entire demand is completely covered:

$\sum_{\mathrm{k}=1}^{\mathrm{N}} \sum_{\mathrm{l}=1}^{\mathrm{N}} \lambda_{\mathrm{kl}}^{\mathrm{n}}=\lambda \quad(\mathrm{n}=1,2, \ldots)$

If unit k's state changes from idle to busy then $B_{i}=\left\{b_{1}, \ldots, 0_{k}, \ldots, b_{N_{-} 1}, b_{N}\right\}$ and $B_{j}=\left\{b_{1}, \ldots, 1_{k}, \ldots\right.$ ., $\left.\mathrm{b}_{\mathrm{N} \_1}, \mathrm{~b}_{\mathrm{N}}\right\}$. The upward transition $\lambda_{\mathrm{ij}}$ can be calculated as follows:

$\lambda_{\mathrm{ij}}=\lambda_{\mathrm{kk}}^{1}+\sum_{\mathrm{l}_{1} \in \mathrm{N}: \mathrm{b}_{\mathrm{l}_{1}=1}} \lambda_{\mathrm{l}_{1} \mathrm{k}}^{2}+\sum_{\mathrm{m}=2}^{\mathrm{M}} \sum_{\mathrm{l}_{1}, \ldots, \mathrm{l}_{\mathrm{m}-1} \in \mathrm{N}: \prod_{\mathrm{i}=1}^{\mathrm{m}-1} \mathrm{~b}_{\mathrm{l}_{\mathrm{i}}}=1} \lambda_{\mathrm{l}_{1} \mathrm{k}}^{\mathrm{m}} \cap \lambda_{\mathrm{l}_{1} \mathrm{l}_{\mathrm{m}-1}}^{\mathrm{m}-1} \cap \ldots \cap \lambda_{\mathrm{l}_{1} \mathrm{l}_{2}}^{2}$

where $M$ is the maximum order of districting ( $M \leq$ number of busy servers).

The calculation of the downward rates $l_{i j}$ is based on the upward rates $k_{i j}$. If Eq. (21A) has $P$ different terms (different sub-areas), denoted for the simplicity as $\lambda_{p}$ and $\mu_{p}$ is the service rate for each of these sub-areas, the downward rate $\mu_{\mathrm{ij}}$ is

$\mu_{\mathrm{ij}}=\frac{\lambda_{\mathrm{ij}}}{\sum_{\mathrm{p}=1}^{\mathrm{P}}\left(\frac{\lambda_{p}}{\mu_{p}}\right)}$

\section{The implementation of the solutions method}

Locating issues, even the simplest, like MCLP model are NP-hard problems. Therefore, the models, which are more complex than MCLP model, are also NP-hard. As a result, the model presented in this study with regard to this topic, which is more complex than the MCLP model is categorized as NP-hard problems. The method of solving such problems is single-pointed innovative. Methods that have been used in this study are simulated annealing (SA), genetic algorithm (GA) and a hybrid of these two methods.

\subsection{Simulated Annealing (SA)}

This method is a discrete optimization technique and the policy of algorithm is inspired from slow freezing process in physics science. This method is an algorithm based on local search that has ability to quit from local optimization, which first by irregular jump and by time passage to reach in a better point trying to have a more systematic search. There are some components for implementation of the algorithm SA such as resolve policy, coding procedure, and production of the initial answer, temperature reduction program and stop criteria. Each of these components will briefly describe.

\subsubsection{Coding procedure}

Coding procedures is a procedure to declare a policy to answer the issue to a computer. Coding procedures in research is in form of zero and one.

\subsubsection{Primary answer production}

We can select primary answer in two ways, random or accept a specified good answer as the primary answer. In this study, way of selection is random. The structure of the answer is a $1 \times \mathrm{J}$ array where $j$ represents the number of demand regions. As was said in the previous section, we may have the 
answer to be understandable by the computer coding. So to create the answer at first, an array of $1 \times$ $(\mathrm{J} \times \mathrm{N})$ is created where $N$ is the number of available ambulances, and ( 0 and 1$)$ components with the condition that the sum of components in the array is equal $N$. Fig. 1 is an example. (It is assumed $\mathrm{N}=$ 3 and $\mathrm{J}=5$ ).

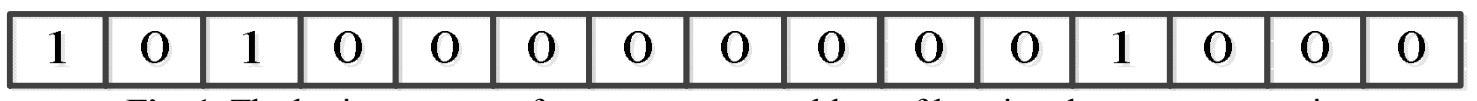

Fig. 1. The basic structure of an answer to a problem of locating the emergency units

Now array is divided by $J \varepsilon N$ cells and fill in new cell. In this way, the answer is coded, finally the final structure can be seen in Fig. 2. This means that each location has few ambulances or facilitate.

\begin{tabular}{|l|l|l|l|l|l|l|l|l|l|l|l|l|l|l|}
\hline $\mathbf{1}$ & 0 & $\mathbf{1}$ & 0 & 0 & 0 & 0 & 0 & 0 & 0 & 0 & 1 & 0 & 0 & 0 \\
\hline
\end{tabular}

Fig. 2 .The final structure for a solution of a hypothetical problem emergency units positioning

\subsubsection{Operator of move}

For the production of a current answer, neighborhood operators of move are used. The structure of the neighborhood determines how to move from one mode to the other mode. In this study, the strategy of creating the neighborhood, which fits the issue is explained next and the implementation is demonstrated in Fig. 3.

The strategy of locating: a random position of the primary structure of the answer is selected.

Alternative strategy: If the selected component is zero instead of one in the same position and zero, otherwise.

Control strategy: the sum of the array components. If the obtained value is less than $N$ locating strategy runs until a position with zero value is selected and the replacement strategy runs on component and if the value obtained is greater than $N$. Therefore, the strategy of locating runs until a position is selected and the component value is one and the replacement strategy runs on that component. After creating the neighborhood, if the result is improved we accept the new result, otherwise, we may accept or decline.

\subsubsection{Temperature reduction program}

Use this tool to determine how to lower the temperature. In this research, in order to lower the temperature, we use linear cold function $T_{l}=T_{(l-1)} \times \alpha$ where $\alpha$ is a positive number less than 1 and called cold rate.

\subsubsection{Stop criteria}

Temperature based stop criteria is considered and as long as the current temperature is greater than one the algorithm goes forward. Although it may decrease the temperature until we reach the desired temperature to stop, it may cause more time to solve algorithm but reaching a favorable answer has greater priority in the research.

\subsection{Genetic Algorithm (GA)}

In the 70's, a scientist from the University of Michigan named John Holland presented the idea of using genetic algorithms in optimization of engineering. The basic idea of the algorithm is inherited characteristics by gene transfer. Components that make the structure of the algorithm are: one issue to resolve, coding procedures and display the answer, production of the initial population, evaluation function, parental choice, Crossover and Mutation operators and stop quality. 


\begin{tabular}{|c|c|c|c|c|c|c|c|c|c|c|c|c|c|c|c|}
\hline \multirow[t]{6}{*}{ Current solution } & 1 & 0 & 1 & 0 & 0 & 0 & 0 & 0 & 0 & 0 & 0 & 1 & 0 & 0 & 0 \\
\hline & 1 & 0 & 1 & 0 & 0 & 0 & 0 & 0 & 0 & 0 & 0 & 1 & 0 & 0 & 0 \\
\hline & 0 & 0 & 1 & 0 & 0 & 0 & 0 & 0 & 0 & 0 & 0 & 1 & 0 & 0 & 0 \\
\hline & 0 & 0 & 1 & 0 & 0 & 0 & 0 & 0 & 0 & 0 & 0 & 1 & 0 & 0 & 0 \\
\hline & 0 & 0 & 1 & 0 & 0 & 0 & 0 & 0 & 0 & 0 & 0 & 1 & 0 & 0 & 0 \\
\hline & 0 & 0 & 1 & 1 & 0 & 0 & 0 & 0 & 0 & 0 & 0 & 1 & 0 & 0 & 0 \\
\hline New solution & 0 & 0 & 1 & 1 & 0 & 0 & 0 & 0 & 0 & 0 & 0 & 1 & 0 & 0 & 0 \\
\hline
\end{tabular}

Fig. 3. Neighborhood create operators, in designed SA algorithm
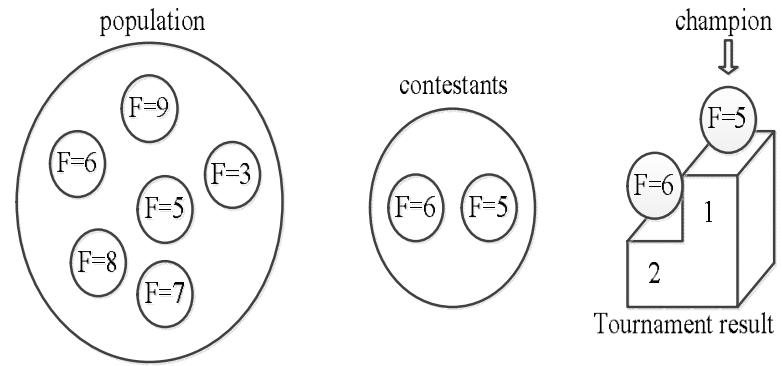

Fig. 4. Schematic picture of Binary Tournament method

\subsubsection{The evaluation function}

By using this function, each chromosome is decoded and a quality and a value is assigned to it.

\subsubsection{Parental choice method}

In this research to choose the parents of existing population, tournament method is a type of 0,1 is used. In this way all the existing answers have the same chance of selection and work started with choosing two random answers from population and one of those is selected based on the amount of fitted as a parent. Fig. 4 shows the proposed GA model,

\subsubsection{Crossover operator}

Crossover operator executes combining two selected parents hoping to create two better new children, to continue the process of the evolution. The crossover operator receives two chromosomes and creates maximum two children. In the study two points method is used. First two random points are selected, all the genes between two points on parents are passed on to children and remained genes in order of existing in other parent will move to other child. Fig. 5 shows details of our method.
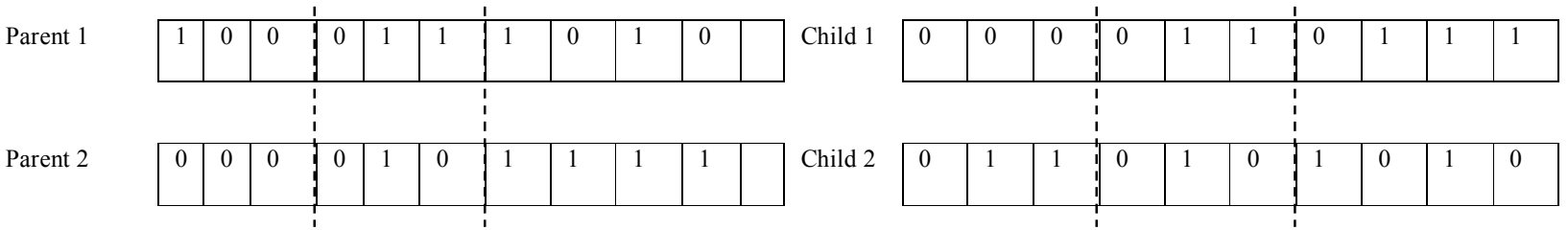

Fig. 5. Schematic view of Two Point Crossover method

\subsubsection{Mutation operator}

In this study, the way of mutation operator is to select a random gene and the inner number of gene is changed, if the number is 0 changes to 1 and 0 to 1 . Now the total numbers within the gene are calculated, if this value is not equal to the number of available ambulances, again we choose a random gene and the number inside it changes with condition. By changing it, the sum of the gene numbers is equal to the number of ambulances, otherwise, we select a different random gene and continue to meet the desired condition.

\subsubsection{Stop criteria}

In order to end the genetic algorithm, several criteria can be used. Here stop criteria is the number of evolved generations.

\subsection{Combined algorithm of $S A$ and $G A$ algorithms}

By combining two or more algorithms, contrasting behavior characteristics are also combined and the performance of established algorithm can be improved. In different compositions, an algorithm can be combined, the most important are serial combination, parallel combination and within 
combination. In this study, we use serial combination, the final answer of genetic algorithm is initial response to simulated refrigeration algorithm. Because the genetic algorithm is not sensitive to initial answers and the final answer is not much influenced by initial answers. On the other hand, we know if refrigeration simulation algorithm works with a good initial solution due to the high escalated ability, certainly will reach to a good answer. Some successful combination of SA \& GA are referred by Roach and Nagi (1996), Ghoshal (2004), Mohanta et al. (2007), Chen and Shahandashti (2009), Oyso and Bingul (2009), Sonmez and Bettemir (2012) and Zameer et al. (2014).

\section{Computational results}

To evaluate the performance of these three algorithms, first, several samples in accordance with the model are created and after setting the parameters, they are solved by algorithms. In order to determine the most appropriate algorithm for solving this group of positioning issues we will compare the results.

\subsection{Creating sample issue}

The parameters required to create sample issues are: The number of regions looking for services, the area required for the establishment of any ambulance, the number of existing ambulances, the minimum acceptable level of coating, the maximum available budget, the coating standard time, the number and the severity of potential events and the population density in each area, the land prices in each area for establishment of ambulance, the time interval between all regions and the weight of each of the target functions. These factors and interval are considered in Table 1.

\section{Table 1}

Sample issues parameters and their limits

\begin{tabular}{|c|c|c|c|}
\hline Factor & region & Factor & region \\
\hline The number of regions calls for the population & {$\left[\begin{array}{ll}9 & 50\end{array}\right]$} & Numbers of probable & {$\left[\begin{array}{ll}1 & 10\end{array}\right]$} \\
\hline Needed area for establishment of any ambulance & {$\left[\begin{array}{ll}1 & 3\end{array}\right]$} & Intensity of probable & {$\left[\begin{array}{ll}1 & 10\end{array}\right]$} \\
\hline Number of existing ambulances & {$\left[\begin{array}{ll}9 & 50\end{array}\right]$} & Population density in each & {$\left[\begin{array}{ll}1 & 10\end{array}\right]$} \\
\hline Minimum acceptable of covering area & {$\left[\begin{array}{ll}.7 & .99\end{array}\right]$} & Land price in each area & {$\left[\begin{array}{ll}30 & 200\end{array}\right]$} \\
\hline Maximum available budget & {$\left[\begin{array}{ll}300 & 2000\end{array}\right]$} & Time interval between & $\left\lceil\begin{array}{ll}1 & 10\end{array}\right]$ \\
\hline Standard covering time & {$\left[\begin{array}{ll}5 & 8\end{array}\right]$} & & \\
\hline
\end{tabular}

\subsection{Parameter setting}

The parameter setting is one of the most important components of an innovative algorithm, which has the major impact on the performance. Table 2 shows refrigeration simulated algorithm input parameters as well as the selected levels. For the suitable set of the algorithm parameters, we run questions five times with five sizes in MATLAB for achieving the lowest objective function and change the results (values of the objective functions) to the relative deviation of the percentage indicator (RPD), the results of MATLAB in accordance with Eq. (22) and Eq. (23) will be converted to this index and finally, using testing design in MINITAB software (version 16) average of resulting RPD checked. By examining Fig. 6 and the Table 3 and analyzing the final results of the software MINITAB we conclude that combination temperature parameter in level 3, the alpha parameter in level 5 and MaxIt parameter in level 2 yield a better performance. In summary, the results can be viewed in the Table 4.

Table 2

Input parameters of refrigeration simulation algorithm

\begin{tabular}{lccc}
\hline Factors & Temperature & Alpha & MaxIt \\
\hline Levels & $50-150$ & $0.8-0.99$ & $20-50$ \\
\hline
\end{tabular}

$$
\begin{aligned}
& \mathrm{RPD}=\frac{\mid \text { Algorithm }_{\text {sol }}-\text { Best }_{\text {sol }} \mid}{\text { Best }_{\text {sol }}} \times 100 \\
& \mathrm{ARPD}=\overline{\mathrm{RPD}}=\frac{\sum_{\mathrm{i}=1}^{\text {Number of run }} \mathrm{RPD}}{\text { Number of run }}
\end{aligned}
$$


Algorith $_{\text {sol }}$ of the amount of objective function for a specific question is specified. Best sol $_{\text {is }}$ the minimum value of the objective function are among the results achieved.

Table 3

RPD total averages for every combination.

\begin{tabular}{|c|c|c|c|c|c|c|c|c|c|}
\hline NO. & temperature & alpha & MaxIt & ARPD & NO. & temperature & alpha & MaxIt & ARPD \\
\hline 1 & 50 & 0.8 & 20 & 2.030411 & 31 & 100 & 0.9 & 40 & 2.545093 \\
\hline 2 & 50 & 0.8 & 30 & 3.150903 & 32 & 100 & 0.9 & 50 & 2.718226 \\
\hline 3 & 50 & 0.8 & 40 & 3.176465 & 33 & 100 & 0.95 & 20 & 2.248257 \\
\hline 4 & 50 & 0.8 & 50 & 1.813668 & 34 & 100 & 0.95 & 30 & 2.551799 \\
\hline 5 & 50 & 0.85 & 20 & 3.662824 & 35 & 100 & 0.95 & 40 & 2.821449 \\
\hline 6 & 50 & 0.85 & 30 & 1.76158 & 36 & 100 & 0.95 & 50 & 1.58458 \\
\hline 7 & 50 & 0.85 & 40 & 2.897191 & 37 & 100 & 0.99 & 20 & 1.557005 \\
\hline 8 & 50 & 0.85 & 50 & 2.312985 & 38 & 100 & 0.99 & 30 & 0.622008 \\
\hline 9 & 50 & 0.9 & 20 & 2.308249 & 39 & 100 & 0.99 & 40 & 1.531592 \\
\hline 10 & 50 & 0.9 & 30 & 1.156827 & 40 & 100 & 0.99 & 50 & 0.987595 \\
\hline 11 & 50 & 0.9 & 40 & 2.11823 & 41 & 150 & 0.8 & 20 & 1.787572 \\
\hline 12 & 50 & 0.9 & 50 & 2.287474 & 42 & 150 & 0.8 & 30 & 2.437263 \\
\hline 13 & 50 & 0.95 & 20 & 1.810775 & 43 & 150 & 0.8 & 40 & 2.888002 \\
\hline 14 & 50 & 0.95 & 30 & 2.222103 & 44 & 150 & 0.8 & 50 & 2.293158 \\
\hline 15 & 50 & 0.95 & 40 & 1.787055 & 45 & 150 & 0.85 & 20 & 2.003632 \\
\hline 16 & 50 & 0.95 & 50 & 1.566903 & 46 & 150 & 0.85 & 30 & 1.849884 \\
\hline 17 & 50 & 0.99 & 20 & 0.990911 & 47 & 150 & 0.85 & 40 & 2.149828 \\
\hline 18 & 50 & 0.99 & 30 & 1.360141 & 48 & 150 & 0.85 & 50 & 2.366179 \\
\hline 19 & 50 & 0.99 & 40 & 0.904622 & 49 & 150 & 0.9 & 20 & 2.568364 \\
\hline 20 & 50 & 0.99 & 50 & 0.913025 & 50 & 150 & 0.9 & 30 & 2.679819 \\
\hline 21 & 100 & 0.8 & 20 & 3.958418 & 51 & 150 & 0.9 & 40 & 2.335867 \\
\hline 22 & 100 & 0.8 & 30 & 2.770093 & 52 & 150 & 0.9 & 50 & 1.620695 \\
\hline 23 & 100 & 0.8 & 40 & 3.38322 & 53 & 150 & 0.95 & 20 & 2.066858 \\
\hline 24 & 100 & 0.8 & 50 & 2.45935 & 54 & 150 & 0.95 & 30 & 1.437718 \\
\hline 25 & 100 & 0.85 & 20 & 1.992338 & 55 & 150 & 0.95 & 40 & 1.597699 \\
\hline 26 & 100 & 0.85 & 30 & 0.516732 & 56 & 150 & 0.95 & 50 & 2.584224 \\
\hline 27 & 100 & 0.85 & 40 & 2.21936 & 57 & 150 & 0.99 & 20 & 1.589379 \\
\hline 28 & 100 & 0.85 & 50 & 2.988631 & 58 & 150 & 0.99 & 30 & 1.782064 \\
\hline 29 & 100 & 0.9 & 20 & 2.328364 & 59 & 150 & 0.99 & 40 & 0.728149 \\
\hline 30 & 100 & 0.9 & 30 & 1.166194 & 60 & 150 & 0.99 & 50 & 0.098507 \\
\hline
\end{tabular}

Table 4

The proposed SA algorithm parameters values

\begin{tabular}{lcc}
\hline MaxIt & Temperature & Alpha \\
\hline 30 & 150 & 0.99 \\
\hline
\end{tabular}

According to the procedure described, innovative genetic algorithm parameters were also set. Genetic algorithm input parameters and levels of choice have been shown in Table 5.

Table 5

The levels of the genetic algorithm parameters

\begin{tabular}{lcccc}
\hline Factor & Mutation & Crossover & nPop & MaxIt \\
\hline Level & $0.8-0.99$ & $0.01-0.2$ & $10-50$ & $10-30$ \\
\hline
\end{tabular}

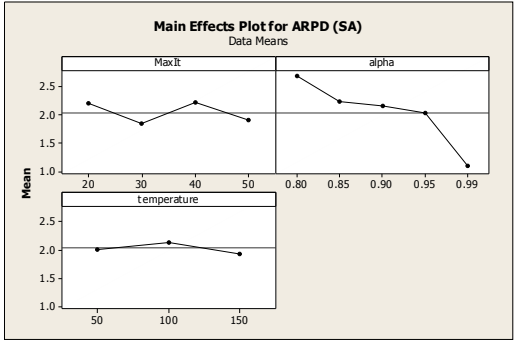

Fig. 6. The achieved results of implementation of software MINITAB for achieved ARPD from running algorithm SA

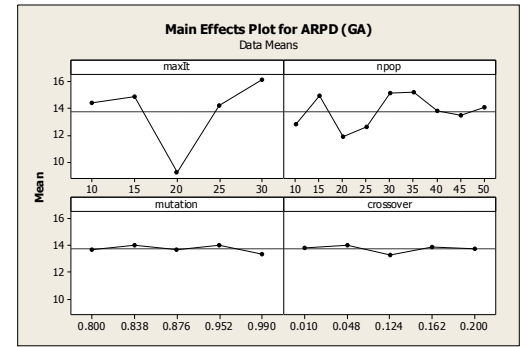

Fig. 7. The MINITAB software implementation results for ARPD made in from GA algorithm

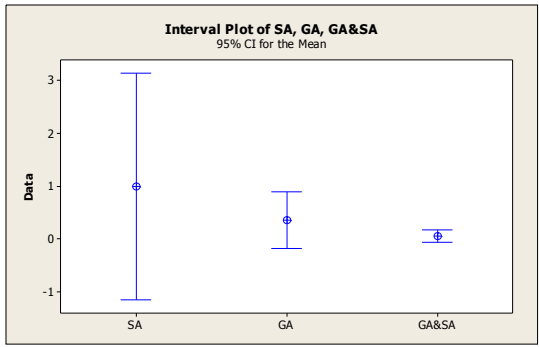

Fig. 8. The scope of an existing algorithm output changes

Finally, with regard to the target function fitting criteria, turn it into a relative deviation percent index and reviews Fig. 7 obtained from MINITAB software, MAXIT parameter in level 3, NPOP parameter in level 3, mutation parameter in level 5 and crossover parameter in level 3 will have best performance. In summary, the results can be found in Table 6. Due to the large volume of results of MATLAB, we ignore. 
Table 6

The GA algorithm proposed parameters values

\begin{tabular}{cccc}
\hline Mutation & Crossover & nPop & MaxIt \\
\hline 0.99 & 0.124 & 20 & 20 \\
\hline
\end{tabular}

\subsection{Comparison of the proposed algorithms with LINGO software}

In order to compare the designed algorithms with LINGO software, we have produced several issues in three small, medium and large sizes and we have solved them by LINGO software. Now any of the above three issues with any of the existing algorithms have been solved and the average solutions have been calculated. To obtain the average relative error percentage we use Eq. (23) and Eq. (24). After the calculation of the relative error percentage for each issue we observe that the maximum relative error for the implementation of the genetic algorithm, simulation of refrigeration algorithm and hybrid algorithm are $1.93683 \%, 0.903348 \%$ and $0.90 \%$, respectively. In addition, the average relative errors for thee mentioned methods are $0.409511 \%, 0.769066 \%$ and $0.405 \%$, respectively, which are very small numbers.

\subsection{Algorithm comparison}

After setting the parameters for the algorithms, sample problems have been solved by all three algorithms in specific time and the results of them (target function fitting) are converted to relative deviation of percentage index and compared with $95 \%$ statistical analysis of trust domain. The result of this analysis and evaluation is displayed in Fig. 8, and it can be seen that hybrid algorithm is better than algorithms and acted with fewer domain changes than the rest.

\section{Discussion and conclusions}

Emergency medical service centers with consideration in a timely manner to patients, decrease mortality and losses due to injuries. Because the goal is to save lives, suggestions to improve the performance of these centers are very welcomed. One of the most important parameters in the provision of emergency medical services with high quality is the time of providing these services and the amount of covering the possible accidents. The location of establishing these centers has a fundamental role for reducing response time to demands and covering these demands and reducing the cost of locating units and hence, the location of these units in cities, especially the big and populated cities is very important. In this study, in order to achieve to the proper place for the establishment of emergency medical services or emergency vehicles, a single objective mathematical model with the aim of minimizing the objective function with constraints including the number of available ambulances, limited budget and minimum acceptable level of covering has been presented. Then three heuristic approaches include SA algorithm and GA algorithm and a hybrid of SA and GA to achieve the optimal or near optimal solution have been used. The preliminary results have indicated that the hybrid algorithm may perform better than two SA and GA algorithms

\section{References}

Ball, M.O., \& Lin, F.L. (1993). A reliability model applied to emergency service vehicle location. Operations Research, 41(1), 18-36.

Burkey, M.L., Bhadury, J., \& Eiselt, H.A. (2012). A location-based comparison of health care services in four U.S. States with efficiency and equity. Socio-Economic Planning Sciences, 46(2), 157-163.

Chen, P.H., \& Shahandashti, S.M. (2009). Hybrid of genetic algorithm and simulated annealing for multiple project scheduling with multiple resource constraints. Automation in Construction, 18(4), 434-443.

Church, R.L., \& ReVelle, C. (1974). The maximal covering location problem. Papers of the Regional Science Association, 32(1), 101-118. 
Daskin, M.S. (1983). A maximum expected covering location model. Formulation, properties and heuristic solution. Transportation Science, (17), 48-70.

Davari, S., Fazel Zarandi, M.H., \& Hemmati, A. (2011) .Maximal covering location problem (MCLP) with fuzzy travel times. Expert Systems with Applications, 38(12), 14535-14541.

Gendreau, M., Laporte, G., \& Semet, F. (1997). Solving an ambulance location model by tabu search. Location Science, 5(2), 75-88.

Geroliminis,N., Karlaftis, M.G., \& Skabardonis, A. (2009). A spatial queuing model for the emergency vehicle districting and location problem. Transportation Research Part B, 43(7), 798-811.

Ghoshal, S.P. (2004). Application of GA/GA-SA based fuzzy automatic generation control of a multi-area thermal generating system. Electric Power Systems Research, 70 (2), 115-127.

Hogan, K., \& ReVelle, C. (1986). Concepts and applications of backup coverage. Management Science, 32(11), 1434-1444.

Holland ,J.H. (1975). Adaptation in Natural and Artificial Systems. University of Michigan Press.

Kirkpatrick, S., Gelatt, C.D., \& Vecchi, M.P. (1983). Optimization by Simulated Annealing. Science, 220(4598), 671-680.

Mohanta, D.K., Sadhu, P.K., \& Chakrabarti, R. (2007). Deterministic and stochastic approach for safety and reliability optimization of captive power plant maintenance scheduling using GA/SA-based hybrid techniques: A comparison of results. Reliability Engineering \& System Safety, 92(2), 187-199.

Oyso, C., \& Bingul, Z. (2009). Application of heuristic and hybrid-GASA algorithms to tool-path optimization problem for minimizing airtime during machining. Engineering Applications of Artificial Intelligence, 22(3), 389-396.

Pirkul, H., \& Schilling, D.A. (1988). The siting of emergency service facilities with workload facilities and backup service. Management Science, 34(7), 896-908.

Repede, J.F., \& Bernardo, J.J. (1994). Developing and validating a decision support system for location emergency medical vehicles in Louisville, Kentucky. European Journal of Operational Research, 75(3), 567-581.

ReVelle, C., \& Hogan, K. (1989). The maximum availability location problem. Transportation Science, 23(3), 192-200.

ReVelle, C. (1989) Review, extension and prediction in emergency services siting models. European Journal of Operational Research, 40(1),58-69.

Roach, A., \& Nagi, R. (1996). A hybrid GA-SA algorithm for just-in-time scheduling of multi-level assemblies. Computers \& Industrial Engineering, 30(4), 1047-1060.

Shariff, S.S.R., Moin, N.H., \& Omar, M. (2012). Location allocation modeling for healthcare facility planning in Malaysia. Computers \& Industrial Engineering, 62(4), 1000-1010.

Schmid, V., \& Doerner, K.F. (2010). Ambulance location and relocation problems with time-dependent travel times. European Journal of Operational Research, 207(3), 1293-1303.

Sonmez, R., \& Bettemir, O.H. (2012). A hybrid genetic algorithm for the discrete time-cost trade-off problem. Expert Systems with Applications, 39(13), 11428-11434.

Syam, S.S., \& Côtéb, M.J. (2010). A location-allocation model for service providers with application to not-for-profit healthcare organizations. Omega, 38(3-4),157-166.

Toregas, C., Swain, R., ReVelle, C., \& Bergman, L. (1971). The location of emergency service facilities. Operations Research, 19(6), 1363-1373.

Weber, A. (1909). Alfred Weber's Theory of the Locaion of Industries: University of Chicago.

Zameer, A., Mirza, S.M., \& Mirza, N.M. (2014). Core loading pattern optimization of a typical two-loop 300 MWe PWR using Simulated Annealing (SA), novel crossover Genetic Algorithms (GA) and hybrid GA(SA) schemes. Annals of Nuclear Energy, 65, 122-131.

Zhang,J., Dong, M., \& Chen, F. F. (2012). A bottleneck Steiner tree based multi-objective location model and intelligent optimization of emergency logistics systems. Robotics and Computer-Integrated Manufacturing, 29(3), 48-55. 